\title{
Polynomials for Proving Termination of Context-Sensitive Rewriting ${ }^{\star}$
}

\author{
Salvador Lucas \\ DSIC, Universidad Politécnica de Valencia \\ Camino de Vera s/n, E-46022 Valencia, Spain \\ slucas@dsic.upv.es
}

\begin{abstract}
We show how to generate well-founded and stable term orderings based on polynomial interpretations over the real numbers. Monotonicity (another usual requirement in termination proofs) can, then, be gradually introduced in the interpretations to deal with different applications. For instance, the dependency pairs method for proving termination of rewriting, and some restrictions of the rewrite relation which are not monotonic in all arguments of the function symbols, can benefit from this approach. The latter is the case for context-sensitive rewriting $(C S R)$, a simple restriction of rewriting which has been proved useful for describing the semantics of several programming languages (e.g., Maude) and analyzing the properties of the corresponding programs. We show how to automatically generate polynomial interpretations over the real numbers for proving termination of $C S R$.
\end{abstract}

Keywords: Programming languages, rewriting, termination.

\section{Introduction}

Context-sensitive rewriting ( $C S R[12$ ) is a simple restriction of rewriting which can be used to analyze termination of programs of programming languages such as Maude, OBJ2, OBJ3, and CafeOBJ (see [13] for further details and examples). In $C S R$, a replacement map $\mu$ discriminates, for each symbol of the signature, the argument positions $\mu(f)$ on which replacements are allowed. This can improve the termination behavior of programs by pruning (all) infinite rewrite sequences.

Example 1. Consider the TRS $\mathcal{R}$ :
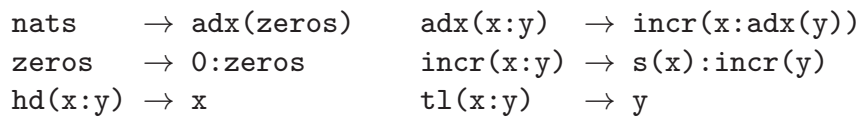

together with $\mu(:)=\mu(\mathrm{s})=\varnothing$ and $\mu($ incr $)=\mu(\operatorname{adx})=\mu($ hd $)=\mu($ tl $)=\{1\}$ [9] Section 1]. Due to $\mu(:)=\varnothing$, the infinite rewrite sequence

$\underline{\text { zeros }} \rightarrow 0$ : $\underline{\text { zeros }} \rightarrow \cdots$

is not possible with $C S R$.

\footnotetext{
* Work partially supported by CICYT TIC2001-2705-C03-01.
} 
Termination of rewriting is undecidable and lot of research has been devoted to develop methods and heuristics to achieve proofs of termination in restricted cases. Polynomial interpretations and the corresponding reduction orderings 11 are well-suited to achieve automatic or semiautomatic proofs of termination of rewriting $36 \mid 1114$. The use of polynomial interpretations has been proposed in [8] as a suitable tool for proving $\mu$-termination, i.e., termination of CSR under a given replacement map $\mu$. In [8], we consider polynomial interpretations consisting of a polynomial $[f]$ on $k$ variables for each $k$-ary symbol $f$, whose coefficients are integers. Such polynomials are actually intended to induce mappings $[f]: \mathbb{N}^{k} \rightarrow \mathbb{N}$ ranging on (and returning) non-negative integers. The polynomial interpretations must also be $\mu$-monotonic, i.e., whenever $x>_{\mathbb{N}} y$, we have $[f]\left(x_{1}, \ldots, x_{i-1}, x, \ldots, x_{k}\right)>_{\mathbb{N}}[f]\left(x_{1}, \ldots, x_{i-1}, y, \ldots, x_{k}\right)$ for all symbols $f \in \mathcal{F}, i \in \mu(f)$ and $x, y, x_{1}, \ldots, x_{k} \in \mathbb{N}$. Then, the interpretation of symbols is homomorphically extended to terms $t$ (where variable symbols are interpreted as variables ranging in $\mathbb{N}$ ) and an ordering $>$ on terms is defined by $t>s$, if $[t]>_{\mathbb{N}}[s]$ for all $x_{1}, \ldots, x_{n} \in \mathbb{N}$, where $x_{1}, \ldots, x_{n}$ are the variables occurring in $t$ or $s$. Now, if $l>r$ for every rule $l \rightarrow r$ of the TRS $\mathcal{R}$, then $\mathcal{R}$ is $\mu$-terminating.

Example 2. Consider the TRS $\mathcal{R}$ :

$$
\begin{array}{ll}
\mathrm{g}(\mathrm{x}) & \rightarrow \mathrm{h}(\mathrm{x}) \quad \mathrm{h}(\mathrm{d}) \rightarrow \mathrm{g}(\mathrm{c}) \\
\mathrm{c} & \rightarrow \mathrm{d}
\end{array}
$$

together with $\mu(\mathrm{g})=\mu(\mathrm{h})=\varnothing[15$, Example 1] (see also [9, Example 16]). By using the results in [8], we prove the $\mu$-termination of $\mathcal{R}$ with the following polynomial interpretation:

$$
\begin{array}{ll}
{[\mathrm{g}](x)=x^{2}-3 x+4} & {[\mathrm{c}]=1} \\
{[\mathrm{~h}](x)=x^{2}-3 x+3} & {[\mathrm{~d}]=0}
\end{array}
$$

The use of negative coefficients in the interpretation is crucial in this example.

The restrictions imposed in [8] for the considered polynomial interpretations are quite usual; moreover, with the early remarkable exception of [5], the polynomials considered in the literature are further restricted to use non-negative integer coefficients $3[11,216]$. This is due to the need to interpret the terms on a well-founded domain (e.g., $\left.\left(\mathbb{N},>_{\mathbb{N}}\right)\right)$. This guarantees that $>$ is a well-founded ordering on terms, hence suitable for termination proofs.

In this paper we show how to overcome these limitations to use more general polynomials which are suitable to, e.g., prove termination of $C S R$.

Example 3. The $\mu$-termination of $\mathcal{R}$ in Example 1 can be proved by using the following polynomial interpretation:

$$
\begin{aligned}
& {[\text { nats }]=9} \\
& {[\operatorname{adx}](x)=x+6} \\
& \text { [zeros] }=2 \\
& \begin{aligned}
{[\operatorname{incr}](x) } & =x+2 \\
{[\mathrm{~s}](x) } & =0 \\
{[\mathrm{hd}](x) } & =2 x+1
\end{aligned} \\
& x[:] y=\frac{1}{2} x+\frac{1}{2} y \\
& \text { [tl] }(x)=2 x+1 \\
& {[0]=0}
\end{aligned}
$$

which, as we will show, can be computed automatically (see Example10 below). 
The framework described in [8] does not apply to polynomial interpretations like that of Example 3 where rational coefficients occur in the polynomials. The main problem is that the set of real (or rational) numbers is not well-founded. Therefore, a term ordering induced by an interpretation mapping terms into, e.g., rational numbers is not guaranteed to be well-founded. On the other hand, the $\mu$-termination of $\mathcal{R}$ in Example 1 cannot be proved within the framework for polynomial termination of $C S R$ given in 8 .

In this paper, we use a general technique to obtain stable and well-founded term orderings by using interpretations of function symbols $f$ as real functions $[f]: \mathbb{R}^{k} \rightarrow \mathbb{R}$ (not necessarily polynomials). Then, we show how to ensure $(\mu-)$ monotonicity of such orderings thus making them $(\mu-)$ reduction orderings and suitable for proving $(\mu-)$ termination of TRSs (Section 3). We discuss how to use this methodology as a basis for proving termination of CSR by means of (more general) polynomial interpretations (Section 4. The framework in 8] can now be seen as a particular case of the new framework. Our extended class of polynomial interpretations provides a powerful tool for proving termination of $C S R$. For instance, all examples of termination of $C S R$ in [9] (the most recent paper on the topic) can be proved now by using our polynomial interpretations. In Section 5, we discuss how to automatically obtain our polynomial interpretations. This is the first time that the automatic generation of direct proofs of termination of $C S R$ is implemented: We have implemented our technique as part of the tool MU-TERM, see

http://www.dsic.upv.es/ slucas/csr/termination/muterm.

\section{Preliminaries}

Let $\mathbb{N}, \mathbb{Z}, \mathbb{Q}$, and $\mathbb{R}$ be the sets of natural, integer, rational and real numbers, respectively; given one of such sets $N$ and $z \in N$, we let $N_{z}=\{x \in N \mid x \geq z\}$ and $N_{>z}=\{x \in N \mid x>z\}$. Given a set $A, \mathcal{P}(A)$ denotes the set of all subsets of $A$. A binary relation $R$ on a set $A$ is terminating if there is no infinite sequence $a_{1} R a_{2} R a_{3} \cdots$. Throughout the paper, $\mathcal{X}$ denotes a countable set of variables and $\mathcal{F}$ denotes a signature, i.e., a set of function symbols $\{f, g, \ldots\}$, each having a fixed arity given by a mapping ar $: \mathcal{F} \rightarrow \mathbb{N}$. The set of terms built from $\mathcal{F}$ and $\mathcal{X}$ is $\mathcal{T}(\mathcal{F}, \mathcal{X})$. Terms are viewed as labelled trees in the usual way. Positions $p, q, \ldots$ are represented by chains of positive natural numbers used to address subterms of $t$. We denote the empty chain by $\Lambda$. Given positions $p, q$, we denote its concatenation as $p . q$. If $p$ is a position, and $Q$ is a set of positions, $p . Q=\{p . q \mid q \in Q\}$. The set of positions of a term $t$ is $\operatorname{Pos}(t)$. The subterm at position $p$ of $t$ is denoted as $\left.t\right|_{p}$ and $t[s]_{p}$ is the term $t$ with the subterm at position $p$ replaced by $s$. The symbol labelling the root of $t$ is denoted as $\operatorname{root}(t)$.

A rewrite rule is an ordered pair $(l, r)$, written $l \rightarrow r$, with $l, r \in \mathcal{T}(\mathcal{F}, \mathcal{X})$, $l \notin \mathcal{X}$ and $\mathcal{V} a r(r) \subseteq \mathcal{V}$ ar $(l)$. The left-hand side $(l h s)$ of the rule is $l$ and $r$ is the right-hand side $(r h s)$. A TRS is a pair $\mathcal{R}=(\mathcal{F}, R)$ where $R$ is a set of rewrite rules. A term $t \in \mathcal{T}\left(\mathcal{F}, \mathcal{X}\right.$ ) rewrites to $s$ (at position $p$ ), written $t \stackrel{p}{\rightarrow}_{\mathcal{R}} s$ (or just 
$t \rightarrow s)$, if $\left.t\right|_{p}=\sigma(l)$ and $s=t[\sigma(r)]_{p}$, for some rule $\rho: l \rightarrow r \in R, p \in \mathcal{P o s}(t)$ and substitution $\sigma$. A TRS is terminating if $\rightarrow$ is terminating.

\subsection{Context-Sensitive Rewriting}

A mapping $\mu: \mathcal{F} \rightarrow \mathcal{P}(\mathbb{N})$ is a replacement map (or $\mathcal{F}$-map) if $\forall f \in \mathcal{F}, \mu(f) \subseteq$ $\{1, \ldots, \operatorname{ar}(f)\}[12]$. The set of $\mu$-replacing positions $\mathcal{P o s}^{\mu}(t)$ of $t \in \mathcal{T}(\mathcal{F}, \mathcal{X})$ is: $\mathcal{P}_{o s}{ }^{\mu}(t)=\{\Lambda\}$, if $t \in \mathcal{X}$ and $\mathcal{P}_{o s}^{\mu}(t)=\{\Lambda\} \cup \bigcup_{i \in \mu(\operatorname{root}(t))} i . \mathcal{P o s}^{\mu}\left(\left.t\right|_{i}\right)$, if $t \notin \mathcal{X}$. In context-sensitive rewriting (CSR [12]), we (only) contract replacing redexes: $t \mu$-rewrites to $s$, written $t \hookrightarrow_{\mu} s$, if $t \stackrel{p}{\rightarrow}_{\mathcal{R}} s$ and $p \in \mathcal{P}_{O} s^{\mu}(t)$.

Example 4. Consider $\mathcal{R}$ and $\mu$ as in Example 1 Then, we have:

hd (zeros) $\hookrightarrow_{\mu}$ hd(0:zeros) $\hookrightarrow_{\mu} 0$

Since $1.2 \notin \mathcal{P}_{\text {os }}{ }^{\mu}$ (hd (0:zeros) $)$, redex zeros cannot be further $\mu$-rewritten in hd $(0:$ zeros $)$.

A TRS $\mathcal{R}$ is $\mu$-terminating if $\hookrightarrow_{\mu}$ is terminating.

\section{Algebras over the Reals and Reduction Orderings}

An ordering $>$ on a set $A$ is well-founded if it admits no infinite chain $a_{1}>$ $a_{2}>\cdots>a_{n}>\cdots$. Given a mapping $f: A^{k} \rightarrow A$ and $i \in\{1, \ldots, k\}$, we say that $>$ is monotonic on the $i$-th argument of $f$ if, whenever $x>y$, we have $f\left(x_{1}, \ldots, x_{i-1}, x, \ldots, x_{k}\right)>f\left(x_{1}, \ldots, x_{i-1}, y, \ldots, x_{k}\right)$ for all $x, y, x_{1}, \ldots, x_{k} \in$ $A$. The problem of proving termination of a TRS is equivalent to finding a wellfounded, stable, and monotonic (strict) ordering $>$ on terms (i.e., a reduction ordering) which is compatible with the rules of the TRS, i.e., such that $l>r$ for all rules $l \rightarrow r$ of the TRS. Here, monotonic means that, for all $k$-ary symbol $f$ and $i \in\{1, \ldots, k\},>$ is monotonic on the $i$-th argument of $f$, when $f$ is viewed as a mapping $f: \mathcal{T}(\mathcal{F}, \mathcal{X})^{k} \rightarrow \mathcal{T}(\mathcal{F}, \mathcal{X})$. Stable means that, whenever $t>s$, we have $\sigma(t)>\sigma(s)$ for all terms $t, s$ and substitutions $\sigma$.

Reduction orderings can be obtained by giving appropriate interpretations to the function symbols of the signature. Given a signature $\mathcal{F}$, an ordered $\mathcal{F}$-algebra, is a triple $\left(A, \mathcal{F}_{A},>_{A}\right)$, where $A$ is a set, $\mathcal{F}_{A}$ is a set of mappings $f_{A}: A^{k} \rightarrow A$ for each $f \in \mathcal{F}$ where $k=\operatorname{ar}(f)$, and $>_{A}$ is a (strict) ordering on $A$. For a given valuation mapping $\alpha: \mathcal{X} \rightarrow A$, the evaluation mapping $[\alpha]: \mathcal{T}(\mathcal{F}, \mathcal{X}) \rightarrow$ $A$ is inductively defined by $[\alpha](x)=\alpha(x)$ if $x \in \mathcal{X}$ and $[\alpha]\left(f\left(t_{1}, \ldots, t_{k}\right)\right)=$ $f_{A}\left([\alpha]\left(t_{1}\right), \ldots,[\alpha]\left(t_{k}\right)\right)$ for $x \in \mathcal{X}, f \in \mathcal{F}, t_{1}, \ldots, t_{k} \in \mathcal{T}(\mathcal{F}, \mathcal{X})$. Then, we can define an ordering $>$ on terms given by $t>s$ if and only $[\alpha](t)>_{A}[\alpha](s)$, for all $\alpha: \mathcal{X} \rightarrow A$. If $>_{A}$ is well-founded, then $>$ also is [16, Section 6.2.1].

In this paper we are interested in using real functions over real numbers to define reduction orderings. We say that $\mathcal{A}=\left(A, \mathcal{F}_{A}\right)$ is an $\mathcal{F}$-algebra over the reals if $A \subseteq \mathbb{R}$. Given $m \in \mathbb{R}$ and $A \subseteq \mathbb{R}$, we say that $f_{A}: A^{k} \rightarrow A$ is $m$-bounded if $f_{A}\left(x_{1}, \ldots, x_{k}\right) \geq m$ for all $x_{1}, \ldots, x_{k} \in A$. If there exists $m \in \mathbb{R}$ such that, $f_{A}$ is $m$-bounded for all $f \in \mathcal{F}$, then we say that $\mathcal{A}=\left(A, \mathcal{F}_{A}\right)$ is $m$-bounded. The 
$\operatorname{order}\left(\mathbb{R}_{m},>_{\mathbb{R}}\right)$ is not well-founded for any $m \in \mathbb{R}$. However, as in [10], given $\delta \in \mathbb{R}_{>0}$, we use the following (strict) ordering on the set of real numbers:

$$
\forall x, y \in \mathbb{R}, x>_{\delta} y \text { if and only if } x-y \geq_{\mathbb{R}} \delta
$$

Thus, we have the following.

Theorem 1. Let $\mathcal{F}$ be a signature, $A \subseteq \mathbb{R}, m \in \mathbb{R}, \mathcal{A}=\left(A, \mathcal{F}_{A}\right)$ be an $m$ bounded $\mathcal{F}$-algebra, and $\delta \in \mathbb{R}_{>0}$. Then, the relation $>_{\delta}$ on terms given by

$$
t>_{\delta} s \Leftrightarrow \forall \alpha: \mathcal{X} \rightarrow A,[\alpha](t)-[\alpha](s) \geq \delta
$$

is a well-founded and stable (strict) ordering on $\mathcal{T}(\mathcal{F}, \mathcal{X})$.

In order to use an ordering $>_{\delta}$ (induced by an $m$-bounded algebra) for proving termination of rewriting, we have to further ensure that $>_{\delta}$ is monotonic. The following example shows the use of Theorem 1 to prove termination of TRSs.

Example 5. Consider the following TRS $\mathcal{R}$ [16, Example 6.2.22]:

$$
f(f(x)) \rightarrow f(g(f(x)))
$$

and the 0-bounded algebra $\left(A, \mathcal{F}_{A}\right)$, where $A=\mathbb{R}_{0}, \mathbf{f}_{A}(x)=\lceil x\rceil+\frac{1}{2}$ and $g_{A}(x)=\lfloor x\rfloor$ (here, $\lceil x\rceil$ is the least integer above -or equal to- $x$ and $\lfloor x\rfloor$ is the integer part of $x)$. Note that $>_{1}$ is monotonic: if $t>_{1} s$, then $[\alpha](t) \geq[\alpha](s)+1$ for all valuations $\alpha: \mathcal{X} \rightarrow A$; hence, since $\lfloor x+1\rfloor=\lfloor x\rfloor+1$ and $\lceil x+1\rceil=\lceil x\rceil+1$, we have $[\alpha](f(t))-[\alpha](f(s))=\lceil[\alpha](t)\rceil-\lceil[\alpha](s)\rceil \geq\lceil[\alpha](s)+1\rceil-\lceil[\alpha](s)\rceil=1$. Similarly, $[\alpha](\mathrm{g}(t))-[\alpha](\mathrm{g}(s))=\lfloor[\alpha](t)\rfloor-\lfloor[\alpha](s)\rfloor \geq\lfloor[\alpha](s)+1\rfloor-\lfloor[\alpha](s)\rfloor=1$. Thus, by Theorem $1>>_{1}$ is a reduction ordering. On the other hand, we have:

$$
\begin{aligned}
{[\alpha](\mathrm{f}(\mathrm{f}(\mathrm{x}))) } & =\left\lceil\lceil\alpha(x)\rceil+\frac{1}{2}\right\rceil+\frac{1}{2}=\lceil\alpha(x)\rceil+\frac{3}{2}, \text { and } \\
{[\alpha](\mathrm{f}(\mathrm{g}(\mathrm{f}(\mathrm{x})))) } & =\left\lceil\left\lfloor\left(\lceil\alpha(x)\rceil+\frac{1}{2}\right)\right\rfloor\right\rceil+\frac{1}{2}=\lceil\alpha(x)\rceil+\frac{1}{2} .
\end{aligned}
$$

Therefore:

$$
[\alpha](\mathrm{f}(\mathrm{f}(\mathrm{x})))-[\alpha](\mathrm{f}(\mathrm{g}(\mathrm{f}(\mathrm{x}))))=\lceil\alpha(x)\rceil+\frac{3}{2}-\left(\lceil\alpha(x)\rceil+\frac{1}{2}\right)=1
$$

Then, $f(f(x))>_{1} f(g(f(x)))$ and $\mathcal{R}$ is terminating.

The following theorem provides a sufficient condition for ensuring monotonicity of $>_{\delta}$ : monotonicity of $>_{\delta}$ in the $i$-th argument of a function symbol $f$ comes when an increment of (at least) $\delta$ in the $i$-th argument of $f_{A}$ increases in (at least) $\delta$ the output of the function $f_{A}$ (for each fixed tuple of arguments).

Theorem 2. Let $\mathcal{F}$ be a signature, $A \subseteq \mathbb{R}, m \in \mathbb{R}$, and $\delta \in \mathbb{R}_{>0}$. Let $\mathcal{A}=\left(A, \mathcal{F}_{A}\right)$ be an m-bounded $\mathcal{F}$-algebra, $f \in \mathcal{F}$ and $1 \leq i \leq \operatorname{ar}(f)$. If $f_{A}$ is differentiable in its $i$-th argument and $\frac{\partial f_{A}\left(x_{1}, \ldots, x_{i}, \ldots, x_{a r(f)}\right)}{\partial x_{i}} \geq 1$, then $>_{\delta}$ is monotonic in the $i$-th argument of $f$.

The following example shows the necessity of requiring $\frac{\partial f_{A}\left(x_{1}, \ldots, x_{i}, \ldots, x_{a r(f)}\right)}{\partial x_{i}} \geq 1$ in Theorem 2 . 
Example 6. Consider the following TRS:

$$
\mathrm{a} \rightarrow \mathrm{c}(\mathrm{a})
$$

Let $A=\mathbb{R}_{0}$. Consider the 0-bounded $\mathcal{F}$-algebra $\left(A,\left\{\mathrm{a}_{A}, \mathrm{c}_{A}\right\}\right)$, where $\mathrm{a}_{A}=2$ and $\mathrm{c}_{A}(x)=\frac{1}{2} x$. According to Theorem 1 the ordering $>_{1}$ induced by this algebra is well-founded and stable. The order, however, is not monotonic in the argument of $\mathrm{c}$ : we have $[\alpha](\mathrm{a})=2$ and $[\alpha](\mathrm{c}(\mathrm{a}))=1$, for all $\alpha: \mathcal{X} \rightarrow A$, i.e.,

$$
\text { a }>_{1} c(a)
$$

However, $[\alpha](\mathrm{c}(\mathrm{c}(\mathrm{a})))=\frac{1}{2}$, i.e., $\mathrm{c}(\mathrm{a}) \ngtr_{1} \mathrm{c}(\mathrm{c}(\mathrm{a}))$. Note that $\frac{\partial \mathrm{c}_{A}}{\partial x}=\frac{1}{2} \geq 1$.

Regarding the choice of $\delta$ when using Theorems 1 and 2 Example 5 also shows that this choice matters: for instance, $>_{\frac{1}{2}}$ is not compatible with $\mathcal{R}$ in Example 5. on the other hand, $>_{\frac{3}{2}}$ is not monotonic. In this paper we will always use $\delta=1$.

\subsection{Polynomial Interpretations}

A monomial in $k$ variables over $\mathbb{R}$ is a function $F: \mathbb{R}^{k} \rightarrow \mathbb{R}$ defined by $F\left(x_{1}, \ldots, x_{k}\right)=a x_{1}^{r_{1}} \cdots x_{k}^{r_{k}}$ for some real number $a \neq 0$ and non-negative integers $r_{1}, \ldots, r_{k}$. The number $a$ is called the coefficient of the monomial; $\sum_{i=1}^{k} r_{i}$ is the degree of the monomial. If $r_{1}=r_{2}=\cdots=r_{k}=0$, then the monomial is called a constant. A polynomial in $k$ variables over $\mathbb{R}$ is the sum of finitely many monomials in $k$ variables over $\mathbb{R}$. The set of polynomials over a set $\left\{x_{1}, \ldots, x_{n}\right\}$ of $n$ distinct variables is denoted by $\mathbb{R}\left[x_{1}, \ldots, x_{n}\right]$.

The use of polynomials in termination proofs is normally restricted to

1. polynomials $P$ with non-negative integer coefficients (i.e., $P \in \mathbb{N}\left[x_{1}, \ldots, x_{n}\right]$, see, e.g., 311/2 16]) or

2. polynomials $P$ with real coefficients which are either zero or greater than or equal to 1 (i.e., $P \in \mathbb{R}_{1}\left[x_{1}, \ldots, x_{n}\right]$, see, e.g., [5[6]14]).

In the first case, well-foundedness of the induced orderings on terms comes for free due to the use of a well-founded domain $\left(\mathbb{N},>_{\mathbb{N}}\right)$; the use of non-negative integer coefficients for the polynomials guarantees monotonicity of the ordering ([2] Section 5.3], [16, Proposition 10]). Given a polynomial interpretation $\left(\mathbb{N}, \mathcal{F}_{\mathbb{N}},>_{\mathbb{N}}\right)$, the corresponding reduction ordering $>$ is given as follows: for $t, s \in \mathcal{T}(\mathcal{F}, \mathcal{X})$ (as usual, we write $[f]$ for the polynomial associated to $f$ ),

$$
t>s \Leftrightarrow \forall x_{i} \in \mathbb{N},[t]>[s]
$$

where $[x]=x$ if $x \in \mathcal{X}$ and $\left[f\left(t_{1}, \ldots, t_{k}\right)\right]=[f]\left(\left[t_{1}\right], \ldots,\left[t_{k}\right]\right)$. Note that (as usual) we write $[t]$ instead of $[\alpha](t)$ since the variables are interpreted as themselves (ranging on numbers) and a universal quantification is assumed for each variable.

Proposition 1. Let $\mathcal{F}$ be a signature and $\mathcal{F}_{\mathbb{N}}$ be a polynomial interpretation such that $[f]: \mathbb{N}^{k} \rightarrow \mathbb{N}$ for each $k$-ary symbol $f \in \mathcal{F}$. Let $>$ be the ordering on terms induced by $\left(\mathbb{N}, \mathcal{F}_{\mathbb{N}},>_{\mathbb{N}}\right)$ and $>_{1}$ be the ordering induced by the 0 -bounded $\operatorname{algebra}\left(\mathbb{N}, \mathcal{F}_{\mathbb{N}}\right)$. Then, $>=>_{1}$. 
Proposition 1 proves that the orderings induced by the polynomial interpretations in the usual sense correspond to the ordering $>_{1}$ induced by the polynomial interpretation viewed as a 0 -bounded algebra over $\mathbb{N}$.

In the second case above, monotonicity is still guaranteed by the use of nonnegative (and $\geq 1$ ) coefficients and well-foundedness is guaranteed (for finite signatures) if the polynomials $P$ in $\mathbb{R}_{1}\left[x_{1}, \ldots, x_{n}\right]$ additionally have the subterm property: $P\left(x_{1}, \ldots, x_{k}\right)>x_{i}$ for all $1 \leq i \leq k$ (see [5]).

\section{Polynomial Interpretations for Context-Sensitive Rewriting}

Termination of CSR is fully captured by the so-called $\mu$-reduction orderings [15], i.e., well-founded, stable orderings $>$ which are $\mu$-monotonic, i.e., for all $f \in \mathcal{F}$ and $i \in \mu(f),>$ is monotonic in the $i$-th argument of $f$. Then, a TRS $\mathcal{R}=(\mathcal{F}, R)$ is $\mu$-terminating if and only if there is a $\mu$-reduction ordering $>$ which is compatible with the rules of $\mathcal{R}$, i.e., for all $l \rightarrow r \in R, l>r$ [15] Proposition 1].

Of course, $\mu$-reduction orderings can also be defined by means of $m$-bounded $\mathcal{F}$-algebras over the reals. Well-foundedness and stability of $>_{\delta}$ is already ensured by Theorem 1. In this sense, Proposition 1 proves that the orderings induced by the polynomial interpretations in $\left[8\right.$ correspond to the ordering $>_{1}$ induced by the polynomial interpretation viewed as a 0 -bounded algebra over $\mathbb{N}$.

Example \%. Consider the following TRS $\mathcal{R}$ :

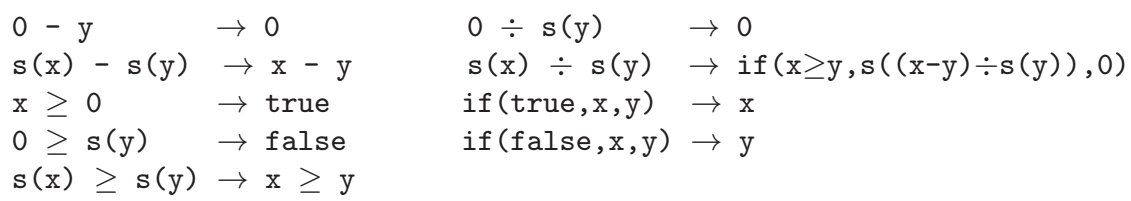

together with $\mu($ if $)=\mu(\div)=\mu(\mathbf{s})=\{1\}$ and $\mu(f)=\varnothing$ for any other symbol $f$ [9] Example 49]. The $\mu$-termination of $\mathcal{R}$ can be proved by using the ordering $>_{1}$ induced by the following polynomial interpretation $\left(\mathbb{N}, \mathcal{F}_{\mathbb{N}}\right)$, where $\mathcal{F}_{\mathbb{N}}$ is:

$$
\begin{array}{rlrl}
x[-] y & =x+1 & {[\text { true }]} & =0 \\
{[0]} & =0 & {[\text { false }]} & =1 \\
{[\mathrm{~s}](x)} & =x+3 & x[\div] y & =x^{2}+x+1 \\
x[\geq] y & =x+2 & {[\text { if }](x, y, z)} & =x z+x+y+1
\end{array}
$$

Our framework strictly includes 8 . For instance, we show that 8 does not suffice for proving $\mu$-termination of $\mathcal{R}$ in Example 1 Note that the polynomial interpretation [:] of ' :' must contain a monomial $a x^{m} y^{n}$ with $m \in \mathbb{N}, a, n \in \mathbb{N}_{1}$; otherwise, the rule $\mathrm{tl}(\mathrm{x}: \mathrm{y}) \rightarrow \mathrm{y}$ cannot be oriented. In this case, however, the rule zeros $\rightarrow 0$ :zeros cannot be oriented. This is managed in our framework by giving ':' a polynomial interpretation whose second (non-replacing) parameter contributes as half of its value. This permits to deal with recursive calls in righthand sides (as in the rule zeros $\rightarrow 0$ :zeros) whilst sufficient information is still kept to be used in left-hand sides (as in the rule $\mathrm{tl}(\mathrm{x}: \mathrm{y}) \rightarrow \mathrm{y}$ ). 
Example 8. Consider the following non-terminating TRS $\mathcal{R}$ :

$$
\begin{array}{ll}
\operatorname{sel}(0, x: y) & \rightarrow \mathrm{x}
\end{array} \quad \quad \text { from }(\mathrm{x}) \rightarrow \mathrm{x}: \operatorname{from}(\mathrm{s}(\mathrm{x}))
$$

and $\mu(:)=\mu(\mathbf{s})=\mu($ from $)=\{1\}$ and $\mu($ sel $)=\{1,2\}$ [12, Example 5]. This TRS can be proved $\mu$-terminating by using the ordering $>_{1}$ induced by the 1-bounded algebra given by $\left(\mathbb{Q}_{1}, \mathcal{F}_{\mathbb{Q}_{1}}\right)$, where $\mathcal{F}_{\mathbb{Q}_{1}}$ is the following polynomial interpretation:

$$
\begin{aligned}
& {[0]=1} \\
& {[\mathbf{s}](x)=2 x} \\
& {[\mathrm{from}](x)=2 x+2} \\
& {[\mathrm{sel}](x, y)=x^{2} y+1} \\
& x[:] y=x+\frac{1}{4} y
\end{aligned}
$$

Note that $>_{1}$ is $\mu$-monotonic: since $x, y \geq 1$, by Theorem 2 we have

$$
\begin{aligned}
& \frac{\partial[\mathbf{s}]}{\partial x}=2 \geq 1 \quad \frac{\partial[\mathbf{s e l}]}{\partial x}=2 x y \geq 1 \quad \frac{\partial[:]}{\partial x}=1 \\
& \frac{\partial[\text { from }]}{\partial x}=2 \geq 1 \quad \frac{\partial[\mathrm{sel}]}{\partial y}=x^{2} \geq 1
\end{aligned}
$$

We finish this section with a last motivating example:

Example 9. Consider the following TRS $\mathcal{R}$ borrowing the well-known Toyama's example:

$$
\begin{aligned}
& c \rightarrow a \\
& c \rightarrow b
\end{aligned} \quad f(a, b, x) \rightarrow f(x, x, x)
$$

together with $\mu(\mathrm{f})=\{1,3\}$. The following 'polynomial' interpretation $\left(\mathbb{Q}_{1}, \mathcal{F}_{\mathbb{Q}_{1}}\right)$, where $\mathcal{F}_{\mathbb{Q}_{1}}$ is:

$$
\begin{aligned}
{[\mathrm{f}](x, y, z) } & =x+x y^{-1}+z y^{-1}+z=\frac{(x y+z)+(x+y z)}{y} & & {[\mathrm{a}]=2 } \\
{[\mathrm{c}] } & =3 & {[\mathrm{~b}] } & =1
\end{aligned}
$$

can be used to formally prove the $\mu$-termination of $\mathcal{R}$. The $\mu$-reduction ordering $>_{1}$ induced by this algebra is compatible with the rules of $\mathcal{R}$ :

$$
\begin{aligned}
{[\mathrm{f}(\mathrm{a}, \mathrm{b}, \mathrm{x})]-[\mathrm{f}(\mathrm{x}, \mathrm{x}, \mathrm{x})] } & =2 x+4-(2 x+2)=2 \geq 1 \\
{[\mathrm{c}]-[\mathrm{a}] } & =3-2=1 \geq 1 \\
{[\mathrm{c}]-[\mathrm{b}] } & =3-1=2 \geq 1
\end{aligned}
$$

Regarding $\mu$-monotonicity of $>_{1}$, we use Theorem 2

$$
\frac{\partial[\mathbf{f}]}{x}=1+y^{-1} \geq 1 \quad \frac{\partial[\mathbf{f}]}{z}=1+y^{-1} \geq 1
$$

We can also prove that $\mathcal{R}$ is simply $\mu$-terminating (see [8 Section 3]): since $x, y, z \geq 1$, we have

$$
\begin{aligned}
& {[\mathrm{f}(\mathrm{x}, \mathrm{y}, \mathrm{z})]-[\mathrm{x}]=x y^{-1}+z y^{-1}+z \geq 1} \\
& {[\mathrm{f}(\mathrm{x}, \mathrm{y}, \mathrm{z})]-[\mathrm{z}]=x+x y^{-1}+z y^{-1} \geq 1}
\end{aligned}
$$

When considering $\mu^{\prime}(f)=\{2,3\}$, the proof of (simple) $\mu^{\prime}$-termination of $\mathcal{R}$ is similar. 


\section{Automatic Proofs of Termination of CSR Using Polynomials}

Polynomial interpretations are well-suited to mechanize the proofs of termination. A proof of termination of a TRS is transformed into the problem of solving a set of constraints over the coefficients of a polynomial interpretation for the symbols of the TRS 11. We are especially interested in proving $\mu$-termination of TRSs by using polynomial interpretations. According to Theorems 1 and 2 the set of constraints is then obtained by requiring the polynomial interpretations to satisfy the following restrictions: for all $f \in \mathcal{F}$,

1. 0-boundedness: $[f]\left(x_{1}, \ldots, x_{k}\right) \geq 0$ for all $x_{1}, \ldots, x_{k} \geq 0$.

2. $\mu$-monotonicity: $\frac{\partial[f]\left(x_{1}, \ldots, x_{i}, \ldots, x_{k}\right)}{\partial x_{i}} \geq 1$ for all $i \in \mu(f)$.

3. Compatibility with the rules of the TRS: $l>_{1} r$ for all $l \rightarrow r \in R$.

For practical reasons, we also restrict the polynomials that we consider to have rational (although possibly negative) coefficients. Thus, $[f] \in \mathbb{Q}\left[x_{1}, \ldots, x_{k}\right]$ and $[f]: \mathbb{Q}_{0}^{k} \rightarrow \mathbb{Q}_{0}$ for each $k$-ary symbol $f \in \mathcal{F}$. This limitation (and other limitations which are introduced below) is motivated by the use of the CiME system [4] to solve the set of constraints that we obtain. CiME is only able to solve Diophantine inequations yielding non-negative integers as solutions. As we will see below, the use of rational numbers is easily made compatible with this limitation. The choice of 0 -bounded polynomial interpretations and that of the ordering $>_{1}$ (i.e., $m=0$ and $\delta=1$ in Theorem [1) is arbitrary.

The following result imposes some general restrictions on the structure of ( $m$-bounded) polynomials containing negative coefficients.

Proposition 2. Let $P \in \mathbb{R}\left[x_{1}, \ldots, x_{k}\right]$ be $m$-bounded for some $m \in \mathbb{R}$ and $a_{r_{1} \cdots r_{k}} x_{1}^{r_{1}} \cdots x_{k}^{r_{k}}$ be a monomial in $P$. If $a_{r_{1} \cdots r_{k}}<0$, then, for all $i \in\{1, \ldots, k\}$ satisfying $r_{i}>0$, there is a monomial $a_{r_{1}^{\prime} \cdots r_{k}^{\prime}} x_{1}^{r_{1}^{\prime}} \cdots x_{k}^{r_{k}^{\prime}}$ in P satisfying $a_{r_{1}^{\prime} \cdots r_{k}^{\prime}}>$ 0 and $r_{i}^{\prime}>r_{i}$.

Thus, if we want to have a negative coefficient for a monomial which is first degree in a given variable $x_{i}$, we need to have a monomial of higher degree in $x_{i}$ having a positive coefficient. Otherwise, $m$-boundedness (which is required in Theorem 11) cannot be achieved.

Of course, the difficulty of the procedure also depends of the complexity of the polynomials that we use for this. In the literature, there are two classes of polynomials which are well-suited for automatization of termination proofs: linear [11] and simple-mixed [14] interpretations. Being the simplest ones, we use linear polynomial interpretations to discuss and exemplify how to proceed for automatically proving termination of CSR by using (or more precisely generating) polynomial interpretations.

\subsection{Using Linear Polynomial Interpretations}

A polynomial $P \in \mathbb{R}\left[x_{1}, \ldots, x_{n}\right]$ is linear, if $P=a_{n} x_{n}+a_{n-1} x_{n-1}+\cdots+a_{1} x_{1}+$ $a_{0}$. According to the previous discussion, we are going to use polynomial interpretations $\left(\mathbb{Q}_{0}, \mathcal{F}_{\mathbb{Q}_{0}}\right)$. Note that, according to Proposition 2 , negative coefficients 
are not allowed in linear polynomial interpretations. Moreover, the independent coefficient $a_{0}$ cannot be negative if $a_{i} \neq 0$ for some $1 \leq i \leq k$; otherwise, the interpretation would not be $m$-bounded for any $m \in \mathbb{R}$.

The following proposition justifies that, for the purpose of proving termination of $C S R$ by using a linear polynomial interpretation inducing an ordering $>_{\delta}$ for some $\delta \in \mathbb{R}_{>0}$, we also have to eventually ensure that, for all $f \in \mathcal{F}$, $0 \leq a_{i}<1$ whenever $i \notin \mu(f)$.

Proposition 3. Let $\mathcal{F}$ be a signature, $A \subseteq \mathbb{R}, \delta \in \mathbb{R}_{>0}$, and $\left(A, \mathcal{F}_{A}\right)$ be a linear polynomial interpretation for $\mathcal{F}$. Then, $>_{\delta}$ is monotonic in the $i$-th argument of $f \in \mathcal{F}$ if and only if $a_{i} \geq 1$, where $[f]=a_{k} x_{k}+a_{k-1} x_{k-1}+\cdots+a_{1} x_{1}+a_{0}$.

Hence, following the previous discussion and results we assume that each $k$-ary symbol $f \in \mathcal{F}$ is interpreted as a linear polynomial $[f]=a_{k} x_{k}+a_{k-1} x_{k-1}+$ $\cdots+a_{1} x_{1}+a_{0}$ where

1. $a_{0} \in \mathbb{N}$,

2. $a_{i} \in \mathbb{N}_{1}$ if $i \in \mu(f)$ and

3. $a_{i}=\frac{p_{i}}{q_{i}}$ if $i \notin \mu(f)$, where $p_{i} \in \mathbb{N}, q_{i} \in \mathbb{N}_{1}$ and $p_{i}<q_{i}$. Here, Proposition 3 is used to adopt an heuristic: since non-replacing arguments are not restricted to be greater than or equal to 1 , we permit the use of rational coefficients $a_{i}$ and, in fact, we impose $0 \leq a_{i}<1$ to (try to) reduce the search space. Note that, in [8], the only possibility would be $a_{i}=0$.

The following example shows how these ideas work in practice: we describe how to prove the polynomial $\mu$-termination of $\mathcal{R}$ in Example 1 .

Example 10. Consider $\mathcal{R}$ and $\mu$ as in Example 1 The symbols of the signature are interpreted as linear polynomials:

$$
\begin{aligned}
{[\operatorname{nats}] } & =a_{0} & {[\mathrm{incr}](x) } & =f_{1} x+f_{0} \\
{[\operatorname{adx}](x) } & =b_{1} x+b_{0} & {[\mathrm{~s}](x) } & =g_{1} x+g_{0} \\
{[\operatorname{zeros}] } & =c_{0} & {[\mathrm{hd}](x) } & =h_{1} x+h_{0} \\
x[:] y & =d_{10} x+d_{01} y+d_{00} & {[\mathrm{tl}](x) } & =i_{1} x+i_{0} \\
{[0] } & =e_{0} & &
\end{aligned}
$$

where, according to Proposition 3, we further assume that $d_{10}=\frac{p_{10}}{q_{10}}$ and $d_{01}=$ $\frac{p_{01}}{q_{01}}$ for natural numbers $p_{10}<q_{10}$ and $p_{01}<q_{01}$. Analogously, for [s] we let $g_{1}=\frac{p_{1}}{q_{1}}$ for natural numbers $p_{1}<q_{1}$. All other coeficients are natural numbers.

Now, we use Theorems 1 and 2 to generate a set of constraints over the unknown coefficients. First we note that, since $\mathrm{C} i \mathrm{ME}$ solves the indeterminate coefficients $a_{i}, b_{i}, \ldots, p_{i}, q_{i}, \ldots$ in $\mathbb{N}$, the 0 -boundedness of each polynomial is immediately guaranteed. Regarding $\mu$-monotonicity and compatibility with the rules of the TRS, we have the following:

1. Constraints due to $\mu$-monotonicity. In general, we use Theorem 2 , here (where linear interpreations are assumed) Proposition 3 can be used instead:
(a) $\frac{\partial[\mathrm{adx}]}{\partial x}=b_{1} \geq 1$
(b) $\frac{\partial[\text { incr }]}{\partial x}=f_{1} \geq 1$
(c) $\frac{\partial[\mathrm{hd}]}{\partial x}=h_{1} \geq 1$
(d) $\frac{\partial[\mathrm{tl}]}{\partial x}=i_{1} \geq 1$ 
2. Constraints due to compatibility with rules (use Theorem 1 with $>_{1}$ ):

a) Compatibility with the rule nats $\rightarrow \operatorname{adx}$ (zeros):

$$
\begin{aligned}
& a_{0}-\left(b_{1} c_{0}+b_{0}\right) \geq 1 \Leftrightarrow \\
& a_{0}-b_{1} c_{0}-b_{0} \geq 1
\end{aligned}
$$

b) Compatibility with the rule zeros $\rightarrow 0:$ zeros:

$$
\begin{aligned}
& c_{0}-d_{10} e_{0}-d_{01} c_{0}-d_{00} \geq 1 \Leftrightarrow \\
& q_{10} q_{01} c_{0}-q_{01} p_{10} e_{0}-q_{10} p_{01} c_{0}-q_{10} q_{01} d_{00} \geq q_{10} q_{01}
\end{aligned}
$$

Note that we have used the definition of $d_{10}$ and $d_{01}$ as rational numbers $d_{10}=\frac{p_{10}}{q_{10}}$ and $d_{01}=\frac{p_{01}}{q_{01}}$ to transform the first constraint into an equivalent one where only the components of the fractions are present.

c) Compatibility with the rule incr $(x: y) \rightarrow s(x): \operatorname{incr}(y)$ :

$$
f_{1}\left(d_{10} x+d_{01} y+d_{00}\right)+f_{0}-\left(d_{10}\left(g_{1} x+g_{0}\right)+d_{01}\left(f_{1} y+f_{0}\right)+d_{00}\right) \geq 1
$$

In these cases, we collect the coefficients accompanying each variable $x_{1}, \ldots, x_{k}$ to obtain a constraint

\begin{tabular}{|c|c|c|}
\hline$q_{1} f_{1} p_{10}-p_{10} p_{1} \geq 0$ & $f_{1} p_{01}-p_{01} f_{1} \geq 0$ & $\wedge$ \\
\hline \multicolumn{3}{|c|}{$q_{10} q_{01} f_{1} d_{00}+q_{10} q_{01} f_{0}-q_{01} p_{10} g_{0}-q_{10} p_{01} f_{0}-q_{10} q_{01} d_{00} \geq q_{10} q_{01}$} \\
\hline
\end{tabular}

$$
A_{k} x_{k}+\cdots+A_{1} x_{1}+A_{0} \geq B
$$

Then, since $x_{1}, \ldots, x_{k} \geq 0$, we express this constraint as

$$
A_{k} \geq 0 \wedge \cdots \wedge A_{1} \geq 0 \wedge A_{0} \geq B
$$

For the constraint above, we obtain

d) Compatibility with the rule $\operatorname{adx}(x: y) \rightarrow \operatorname{incr}(x: \operatorname{adx}(y))$ :

$$
\begin{aligned}
& b_{1}\left(d_{10} x+d_{01} y+d_{00}\right)+b_{0}-\left(f_{1}\left(d_{10} x+d_{01}\left(b_{1} y+b_{0}\right)+d_{00}\right)+f_{0}\right) \geq 1 \Leftrightarrow \\
& b_{1} p_{10}-f_{1} p_{10} \geq 0 \wedge \wedge \\
& q_{01} b_{1} d_{00}+q_{01} b_{0}-f_{1} p_{01} b_{0}-q_{01} f_{1} d_{00}-q_{01} f_{0} \geq q_{01}
\end{aligned}
$$

e) Compatibility with the rule $\mathrm{hd}(\mathrm{x}: \mathrm{y}) \rightarrow \mathrm{x}:$

$$
\begin{aligned}
& h_{1}\left(d_{10} x+d_{01} y+d_{00}\right)+h_{0}-x \geq 1 \Leftrightarrow \\
& h_{1} p_{10}-q_{10} \geq 0 \wedge h_{1} p_{01} \geq 0 \wedge h_{1} d_{00}+h_{0} \geq 1
\end{aligned}
$$

f) Compatibility with the rule $\mathrm{tl}(\mathrm{x}: \mathrm{y}) \rightarrow \mathrm{y}$ :

$$
\begin{aligned}
& i_{1}\left(d_{10} x+d_{01} y+d_{00}\right)+i_{0}-y \geq 1 \Leftrightarrow \\
& i_{1} p_{10} \geq 0 \wedge \quad i_{1} p_{01}-q_{01} \geq 0 \wedge \quad i_{1} d_{00}+i_{0} \geq 1
\end{aligned}
$$


3. Constraints due to the rational coeficients
(a) $q_{10}>p_{10}$
(b) $q_{01}>p_{01}$
(c) $q_{1}>p_{1}$

Now, this set of constraints can be solved as a set of Diophantine inequations using the CiME system. We (automatically) obtain the solution of this set of constraints, thus yielding the following polynomial interpretation 1 :

$$
\begin{aligned}
& {[\text { nats }]=9} \\
& {[\operatorname{adx}](x)=x+6} \\
& \text { [incr }](x)=x+2 \\
& {[\mathrm{~s}](x)=0} \\
& \text { [zeros] }=2 \\
& \text { [hd] }(x)=2 x+1 \\
& \begin{aligned}
x[:] y & =\frac{1}{2} x+\frac{1}{2} y \\
{[\mathrm{tl}](x) } & =2 x+1
\end{aligned} \\
& {[0]=0}
\end{aligned}
$$

which proves the $\mu$-termination of $\mathcal{R}$.

We have implemented our technique as part of the tool MU-TERM. MU-TERM uses $\mathrm{C} i \mathrm{ME}$ as an external tool for solving the Diophantine inequations obtained with our technique. The polynomial interpretation in Example 10 can be automatically obtained with MU-TERM. We refer the reader to the WWW site of MU-TERM for obtaining further details about it.

\subsection{Simple-Mixed Polynomial Interpretations}

A polynomial $P \in \mathbb{R}\left[x_{1}, \ldots, x_{n}\right]$ is simple-mixed iff all exponents are not greater than 1 or $n=1$ and $P=a_{2} x_{1}^{2}+a_{0}$ [14]. Note that, according to Proposition 2 and the discussion following it in Section 5.1, simple-mixed polynomials used in our interpretations cannot contain negative coefficients. The following example shows the use of simple-mixed polynomial interpretations.

Example 11. Consider the following TRS $\mathcal{R}$ [15, Example 5]:

$$
\begin{aligned}
& \text { if }(\text { true }, \mathrm{x}, \mathrm{y}) \rightarrow \mathrm{x} \\
& \text { if }(\mathrm{false}, \mathrm{x}, \mathrm{y}) \rightarrow \mathrm{y}(\mathrm{x}) \rightarrow \text { if }(\mathrm{x}, \mathrm{c}, \mathrm{f}(\text { true }) \text { ) }
\end{aligned}
$$

together with $\mu(\mathrm{f})=\{1\}$ and $\mu($ if $)=\{1,2\}$. Now we first conjecture a simplemixed polynomial interpretation $\left(\mathbb{Q}_{0}, \mathcal{F}_{\mathbb{Q}_{0}}\right)$ for $\mathcal{F}$. Again, we can use MU-TERM to automatically prove the $\mu$-termination of $\mathcal{R}$. We obtain:

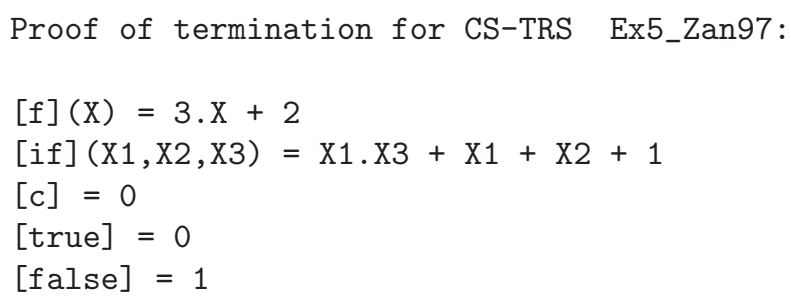

Now we prove that linear interpretations do not work in this case. Assume that there is a linear interpretation $\left(\mathbb{Q}_{0}, \mathcal{F}_{\mathbb{Q}_{0}}\right)$ and some $\delta>0$ such that $>_{\delta}$ is a $\mu$-reduction ordering which is compatible with the rules of $\mathcal{R}$. Such an interpretation includes a polynomial $[$ if $](x, y, z)=a x+b y+c z+d$ interpreting if,

\footnotetext{
${ }^{1}$ We use version 2.0 of the CiME system.
} 
where $a, b \geq 1$ to guarantee that $>_{\delta}$ is $\mu$-monotonic for any $\delta>0$ (Proposition 3). Moreover, $c \geq 1$; otherwise, compatibility of $>_{\delta}$ with the second rule for if would be impossible. Also, the interpretation of $\mathrm{f}$ would be $[\mathrm{f}](x)=m x+n$, where $m \geq 1$ (due to $\mu(\mathrm{f})=\{1\}$ ). Then, by Proposition $3>_{\delta}$ would be, in fact, monotonic, hence a reduction ordering which is compatible with the rules of $\mathcal{R}$. However, being $\mathcal{R}$ non-terminating, no reduction ordering can be compatible with the rules of $\mathcal{R}$.

Example 11 also shows that (in contrast to the usual approaches which use $\mathbb{N}_{1}$ (or $\mathbb{N}_{2}$ ) and $\mathbb{R}_{1}$, see 31114 ] ) the use of 0 for interpreting symbols is crucial for some applications. This claim is justified by the following proposition.

Proposition 4. Let $\mathcal{F}$ be a signature, $A \subseteq \mathbb{R}_{1}, \delta \in \mathbb{R}_{>0}$, and $\left(A, \mathcal{F}_{A}\right)$ be a polynomial interpretation for $\mathcal{F}$. If $[f]$ contains a monomial a $x_{1}^{r_{1}} \cdots x_{i}^{r_{i}} \cdots x_{k}^{r_{k}}$ with $r_{i} \geq 1$ and $a \geq 1$, then, $>_{\delta}$ is monotonic in the $i$-th argument of $f \in \mathcal{F}$.

For instance, without interpreting true as 0 , the $\mu$-termination of $\mathcal{R}$ in Example 11 cannot be proved by using the same interpretations for $f$ and if.

\subsection{Polynomials with Negative Coefficients}

The polynomials in the previous sections do not admit negative coefficients in any monomial. According to Proposition 2, if we want to use negative coefficients in some monomials (as, e.g., in Example 21), we have to consider, at least, the following class of polynomials.

Definition 1 (2-simple-mixed polynomial). A polynomial $P \in \mathbb{R}\left[x_{1}, \ldots\right.$, $\left.x_{n}\right]$ is 2-simple-mixed iff each monomial $a_{r_{1} \cdots r_{k}} x_{1}^{r_{1}} \cdots x_{k}^{r_{k}}$ satisfy either:

1. $r_{i} \in\{0,1\}$ for all $i \in\{1, \ldots, k\}$ or

2. $r_{i}=2$ for some $i \in\{1, \ldots, k\}$ and $r_{j}=0$ for all $j \in\{1, \ldots, k\}-\{i\}$.

Note that simple-mixed polynomials are also 2 -simple mixed. The polynomials used in Example 2 are 2-simple mixed. The following result can be used to guarantee 0-boundedness of a quadratic polynomial:

Proposition 5. Let $P(x)=A x^{2}+B x+C$. Then, $P(x) \geq 0$ for all $x \geq 0$ if and only if either

1. $A \geq 0 \wedge B \geq 0 \wedge C \geq 0$ or

2. $A>0 \wedge B<0 \wedge 4 A C-B^{2} \geq 0$.

Example 12. Consider the following TRS $\mathcal{R}$ :

$$
\begin{array}{lll}
\mathrm{f}(\mathrm{x}, \mathrm{g}(\mathrm{x}), \mathrm{y}) & \rightarrow \mathrm{f}(\mathrm{y}, \mathrm{y}, \mathrm{y}) \quad \mathrm{b} \rightarrow \mathrm{c} \\
\mathrm{g}(\mathrm{b}) & \rightarrow \mathrm{c}
\end{array}
$$

together with $\mu(\mathrm{f})=\{3\}$ [9] Example 24]. The $\mu$-termination of $\mathcal{R}$ can be proved by using the ordering $>_{1}$ induced by:

$$
\begin{aligned}
{[\mathrm{f}](x, y, z) } & =x^{2}-2 x y+y^{2}+z & & {[\mathrm{~b}]=1 } \\
{[\mathrm{~g}](x) } & =x+1 & & {[\mathrm{c}]=0 }
\end{aligned}
$$

In the near future, we plan to furnish MU-TERM with the ability to automatically generate proofs of $\mu$-termination by using polynomials with negative coefficients. 


\section{Conclusions}

We have shown how to obtain stable and well-founded term orderings by using a given interpretation of function symbols as real functions, not necessarily polynomials (Theorem 1). We have shown how to further obtain $(\mu$-)reduction orderings, which can be used for proving ( $\mu$-)termination of TRSs (Theorem 2). This provides a new technique for proving termination of rewriting (Example 5) and a basis for proving termination of $C S R$ by means of very general polynomial interpretations which can be characterized by:

1. The use of rational coefficients (as in Examples 3 and 8),

2. The use of negative coefficients (as in Example 2), and

3. The use of negative exponent:2 (as in Example 9).

These mechanisms allow us to avoid monotonicity for the non-replacing arguments of symbols (where this is not necessary). We also stress that the use of rational, negative coefficients, and negative exponents (and the lack of monotonicity in some arguments) disallows the use of most of the standard results for guaranteeing well-foundedness of the induced ordering on terms. For instance, in contrast to the unrestricted case (see [5]), the $\mu$-subterm property [8] Definition 2] does not guarantee well-foundedness of a term ordering [8 Example 3]. Fortunately, Theorems 1 and 2 provide a good framework for defining $\mu$-reduction orderings.

Regarding their use in proofs of termination of (unrestricted) rewriting, the methods presented here can also be helfpul when monotonicity is not a crucial requirement for the use of term orderings. This is the case of the dependency pairs method, [1] where non-monotonic (but well-founded and stable) orderings can be used in proofs of termination as part of a reduction pair (see [7]).

The framework in 8, can now be seen as a particular case of the new framework (Proposition 1). Our extended class of polynomial interpretations provides quite a powerful tool for proving termination of $C S R$. For instance, all examples of termination of CSR in [9] (the most recent paper on the topic) have been proved terminating now by using polynomial interpretations (see Examples 2 . [3 7 [12, and [8, Example 10]). We have described how to automatically obtain our polynomial interpretations. We have implemented our techniques (linear and simple-mixed interpretations of Sections 5.1 and 5.2) as part of the tool MU-TERM.

\subsection{Future Work}

From a theoretical point of view, the class of reduction orderings $>_{\delta}$ induced by an $m$-bounded algebra over the reals should be further investigated. The exact role of $m$ and $\delta$ has to be clarified. Another interesting question concerns completeness of the approach: is every terminating TRS compatible with one of such orderings? If not, what are the limitations of the approach? Example

\footnotetext{
${ }^{2}$ Of course, we should more properly speak about polynomial fractions in this case.
} 
5 shows that the technique applies to a non-simply terminating TRS (see [16. Proposition 6.3.26(iv)]); thus, what would be the position of these orderings in a termination hierarchy possibly extending that of [16, Section 6.3]?

Regarding the practical use of these orderings, we plan to investigate new families of real functions which are well-suited for automatization purposes. We will focus on those functions which can also be used to prove termination of $C S R$, by introducing mechanisms for loosing monotonicity in some arguments. In particular, in this paper we did not pay any attention to formalize the use of polynomial fractions. This could be a first starting point.

Acknowledgements. I thank Bernhard Gramlich and the anonymous referees for their comments and suggestions.

\section{References}

1. T. Arts and J. Giesl. Termination of Term Rewriting Using Dependency Pairs Theoretical Computer Science, 236:133-178, 2000.

2. F. Baader and T. Nipkow. Term Rewriting and All That. CUP, 1998.

3. A. ben Cherifa and P. Lescanne. Termination of rewriting systems by polynomial interpretations and its implementation. Science of Computer Programming, 9(2):137-160, 1987.

4. E. Contejean and C. Marché. CiME: Completion Modulo E. In Proc. of RTA'96, LNCS 1103:416-419, Springer-Verlag, Berlin, 1996.

5. N. Dershowitz. Orderings for term rewriting systems. Theoretical Computer Science, 17(3):279-301, 1982.

6. J. Giesl. Generating Polynomial Orderings for Termination Proofs. In Proc. of RTA'95, LNCS 914:426-431, Springer-Verlag, Berlin, 1995.

7. J. Giesl, T. Arts, and E. Ohlebusch Modular Termination Proofs for Rewriting Using Dependency Pairs. Journal of Symbolic Computation 38:21-58, 2002.

8. B. Gramlich and S. Lucas. Simple termination of context-sensitive rewriting. In Proc. of RULE'02, pages 29-41, ACM Press, New York, 2002.

9. J. Giesl and A. Middeldorp. Transformation Techniques for Context-Sensitive Rewrite Systems. Journal of Functional Programming, to appear, 2004.

10. D. Hofbauer. Termination Proofs by Context-Dependent Interpretations. In Proc. of RTA'01, LNCS 2051:108-121. Springer-Verlag, Berlin, 2001.

11. D.S. Lankford. On proving term rewriting systems are noetherian. Technical Report, Louisiana Technological University, Ruston, LA, 1979.

12. S. Lucas. Context-sensitive computations in functional and functional logic programs. Journal of Functional and Logic Programming, 1998(1):1-61, January 1998.

13. S. Lucas. Termination of programs with strategy annotations. Technical Report DSIC II/20/03, Universidad Politécnica de Valencia, Sep. 2003.

14. J. Steinbach. Generating Polynomial Orderings. Information Processing Letters 49:85-93, 1994.

15. H. Zantema. Termination of Context-Sensitive Rewriting. In Proc. of RTA'97, LNCS 1232:172-186, Springer-Verlag, Berlin, 1997.

16. H. Zantema. Termination. In TeReSe, editor, Term Rewriting Systems, chapter 6. Cambridge University Press, 2003. 